\title{
CORRECTION OF SOME MISPRINTS IN OUR PAPER*
}

\begin{tabular}{|c|c|c|c|}
\hline Page & Line & Read & Instead of \\
\hline 74 & 7 & $1 / \ln \ln m$; further & $1 / \ln \ln m$. Further \\
\hline 75 & 11 & $2 \sqrt{\ln n} \delta_{n}$ & $\sqrt{\ln n} \delta_{n}$ \\
\hline 75 & 21 & $\geqq\left|\omega\left(\bar{z}_{z}\right)\right|$ & $\geqq \omega\left(\bar{z}_{k}\right)$ \\
\hline 76 & 13 & Dropping $J_{t}$ & Dropping $J_{i}$ \\
\hline 79 & 3 & $\left(n \geqq n_{1}(q)\right)$ & $\left(n \geqq n_{0}(q)\right)$ \\
\hline 80 & 7 & $\sum_{i=1}^{s} f\left(x_{k_{i}}\right) l_{k_{i}}(x)$ & $\sum_{i=1}^{n} f\left(x_{k_{s}}\right) l_{k_{s}}(x)$ \\
\hline 81 & $(4.29)$ & $\sum_{t=1}^{\infty} \cdots$ & $\sum_{t=k}^{\infty} \cdots$ \\
\hline 83 & 24 & that is & and that \\
\hline 86 & $(4.57)$ & $W=\bigcap_{k=1}^{\infty} \bigcup_{t=k} \ldots$ & $W=\bigcup_{k=1}^{\infty} \bigcup_{t=k}^{\infty} \ldots$ \\
\hline 87 & 5 & by $W_{t i}=R_{t i}^{[0]}, \quad W=G^{[0]}$ & by $W=G^{[0]}$ \\
\hline 87 & 6 & $G_{\varrho} \cup W$ & $G_{o} \cap W$ \\
\hline 88 & 3 & $\varepsilon$ and $M(M \geqq 1$, integer $)$ & $\varepsilon$ and $M$ \\
\hline 88 & 10 & $\mu\left(\bigcap_{t=0}^{\infty} H_{t}\right) \geqq \varepsilon, \quad$ which & $\mu\left(\bigcup_{t=0}^{\infty} H_{t}\right) \geqq \varepsilon$ wich \\
\hline 88 & 15 & & \\
\hline
\end{tabular}

Read:

" $\left|L_{u_{1}(x)}\left(f_{1}, x\right)\right| \geqq A_{1}>1^{3} \lambda_{N_{0}}^{2}$ whenever $x \in S_{1}$.

Here $m_{1} \leqq u_{1}(x) \leqq n_{1}$. Now we take the polynomial $\varphi_{1}\left(f_{1}, x\right)$ of degree $\leqq N_{1}$ $\left\|\varphi_{1}\right\| \leqq 32$, for which

(see 4.4.4)."

$$
\left|L_{u_{1}(x)}\left(\varphi_{1}, x\right)\right| \geqq A_{1}>1^{3} \lambda_{N_{0}}^{2} \text { whenever } x \in S_{1}
$$

(see 4.4.4)."

instead of:

$$
{ }^{c}\left|L_{u_{1}(x)}\left(\varphi_{1}, x\right)\right| \geqq A_{1}>1^{3} \lambda_{N_{0}}^{2} \quad \text { whenever } \quad x \in S_{1}
$$

$$
\begin{array}{l|l|l|l}
88 & 16 & \varrho_{k}=2^{-k}, \quad A_{k}=k^{3} \lambda_{N_{k-1}}^{2} & \delta_{k}=2^{-k}, A_{k}>k_{\varepsilon} \lambda_{N_{k-1}}^{2} \\
88 & 18 & \geqq 2-2 \varrho_{k} & \geqq 2-2 \delta_{k} \\
88 & 23 & S=\bigcap_{k=1}^{\infty} \bigcup_{i=k}^{\infty} S_{i} & S=\bigcap_{k=1}^{\infty} \bigcap_{i=k}^{\infty} S_{i}
\end{array}
$$

* P. ERDốs and P. VÉRTESI, On the almost everywhere divergence of Lagrange interpolatory polynomials for arbitrary system of nodes, Acta Math. Acad. Sci. Hungar., 36 (1980), 71-89. 